\title{
A network embedding-based multiple information integration method for the MiRNA-disease association prediction
}

\author{
Yuchong Gong ${ }^{1}$, Yanqing $\mathrm{Niu}^{2}$, Wen Zhang $^{3 *}$ and Xiaohong $\mathrm{Li}^{1^{*}}$
}

\begin{abstract}
Background: MiRNAs play significant roles in many fundamental and important biological processes, and predicting potential miRNA-disease associations makes contributions to understanding the molecular mechanism of human diseases. Existing state-of-the-art methods make use of miRNA-target associations, miRNA-family associations, miRNA functional similarity, disease semantic similarity and known miRNA-disease associations, but the known miRNA-disease associations are not well exploited.

Results: In this paper, a network embedding-based multiple information integration method (NEMII) is proposed for the miRNA-disease association prediction. First, known miRNA-disease associations are formulated as a bipartite network, and the network embedding method Structural Deep Network Embedding (SDNE) is adopted to learn embeddings of nodes in the bipartite network. Second, the embedding representations of miRNAs and diseases are combined with biological features about miRNAs and diseases (miRNA-family associations and disease semantic similarities) to represent miRNA-disease pairs. Third, the prediction models are constructed based on the miRNAdisease pairs by using the random forest. In computational experiments, NEMII achieves high-accuracy performances and outperforms other state-of-the-art methods: GRNMF, NTSMDA and PBMDA. The usefulness of NEMII is further validated by case studies. The studies demonstrate the great potential of network embedding method for the miRNAdisease association prediction, and SDNE outperforms other popular network embedding methods: DeepWalk, HighOrder Proximity preserved Embedding (HOPE) and Laplacian Eigenmaps (LE).

Conclusion: We propose a new method, named NEMII, for predicting miRNA-disease associations, which has great potential to benefit the field of miRNA-disease association prediction.
\end{abstract}

Keywords: miRNA-disease associations, Network embedding, Random forest

\section{Background}

MiRNAs are a kind of small non-coding RNA molecules containing about 22 nucleotides, which are involved in the regulation of post-transcriptional gene expression in plants and animals [1]. MiRNAs are usually considered as negative gene regulators, which regulate the expression of messenger RNAs in a sequence-specific manner and repress the protein translation of their target genes. However, studies showed that miRNAs also act as positive regulators. For example, two well-studied miRNAs:

\footnotetext{
* Correspondence: zhangwen@mail.hzau.edu.cn; leexh@whu.edu.cn ${ }^{3}$ College of Informatics, Huazhong Agricultural University, Wuhan 430070, China

'School of Computer Science, Wuhan University, Wuhan 430072, China Full list of author information is available at the end of the article
}

Let-7 and the synthetic miRNA miRcxcr4 induce translation upregulation of target messenger RNAs on cell cycle arrest [2]. The increasing evidence demonstrated that miRNAs play critical roles in important biological processes, such as cell growth [3], tissue differentiation [4], cell proliferation [5], embryonic development and apoptosis [6, 7]. More importantly, plenty of miRNAs have been discovered to be related to a wide range of human diseases, such as breast cancer, heart diseases and cardiovascular disease [8-10]. Therefore, the identification of miRNA-disease associations is significant for understanding the molecular mechanisms of human diseases and promoting the diagnosis and treatment of human diseases. Experimental determination of miRNA-disease associations is tremendously expensive and laborious, and has a

(c) The Author(s). 2019 Open Access This article is distributed under the terms of the Creative Commons Attribution 4.0 International License (http://creativecommons.org/licenses/by/4.0/), which permits unrestricted use, distribution, and 
high failure rate. Therefore, identifying miRNA-disease associations through computational approaches attracts wide attention from scientific communities.

In the past few years, plenty of computational methods have been developed to predict miRNA-disease associations. For example, Sun et al. [11] proposed a method named NTSMDA, which used the network topological information and the network-based resource allocation algorithm to predict miRNA-disease associations. You et al. [12] constructed a heterogeneous graph by integrating miRNA-disease associations, miRNA-miRNA similarities and disease-disease similarities, and developed a network path-based computational method. Chen et al. [13] proposed a method called RKNNMDA, which implemented k-nearest-neighbor algorithm to select candidate miRNAs (or diseases) and used ranking support vector machine (SVM) to rank candidates and make predictions. Xiao et al. [14] proposed a graph regularized non-negative matrix factorization method called GRNMF, which integrated the disease semantic information, miRNA functional information and miRNA-disease associations. Chen et al. [15] proposed an inductive matrix completion method named IMCMDA by integrating miRNA functional similarity, disease semantic similarity and Gaussian interaction profile kernel similarity. Luo et al. [16] proposed a novel semi-supervised prediction method named MDAGRF based on the graph regularization framework. Chen et al. [17] proposed a bipartite network projection-based method named BNPMDA based on the rating-integrated bipartite network recommendation and the known miRNA-disease associations.

Existing state-of-the-art methods make use of miRNAtarget associations, miRNA-family associations, miRNA functional similarity, disease semantic similarity and known miRNA-disease associations. However, the known miRNAdisease associations are not well exploited. To the best of our knowledge, known miRNA-disease associations can be formed as a bipartite network, but features from the network are seldom considered. The network embedding is to learn embedding representations of nodes by preserving the property of the network. Recently, the network embedding methods, such as DeepWalk [18] and node2vec [19], have been applied to many bioinformatics problems and produced good performances. For example, Zong et al. [20] utilized node embeddings learned by DeepWalk in a heterogeneous network to calculate drug-drug similarity and target-target similarity, and then predicted novel drug-target associations. Li et al. [21] proposed a similarity-based miRNA-disease prediction method, which used DeepWalk to obtain node embeddings and then calculated cosine similarities. Liu et al. [22] used node2vec to obtain node embeddings, and then utilized them to train random forest model for protein complexes identification.
In this paper, a network embedding-based multiple information integration method (NEMII) is proposed for the miRNA-disease association prediction. First, known miRNA-disease associations are formulated as a bipartite network, and the network embedding method Structural Deep Network Embedding (SDNE) is adopted to learn node embeddings in the bipartite network. Second, the embedding representations of miRNAs and diseases are combined with biological features about miRNAs and diseases to represent miRNA-disease pairs. Third, prediction models are constructed based on the miRNAdisease pairs by using random forest. In computational experiments, NEMII achieves high-accuracy performances and outperforms other state-of-the-art methods: GRNMF, NTSMDA and PBMDA. The usefulness of NEMII is further validated by case studies. The studies demonstrate the great potential of network embedding methods for the miRNA-disease association prediction, and the embedding method SDNE outperforms other popular network embedding methods: DeepWalk, HighOrder Proximity preserved Embedding (HOPE) and Laplacian Eigenmaps (LE).

\section{Results \\ Evaluation metrics}

In a miRNA-disease bipartite network, non-association miRNA-disease pairs are much more than association pairs. The miRNA-disease association prediction is a semi-supervised learning task, and the key point is to predict undiscovered miRNA-disease associations from all non-association miRNA-disease pairs. We adopt fivefold cross-validation to evaluate the performances of prediction models. Additional file 1: Figure S1 shows how to implement five-fold cross-validation. The known miRNA-disease associations are randomly equally divided into five subsets. In each fold, one subset of associations is removed, and we can train a prediction model only based on the remaining four subsets of associations. In the stage of training, SDNE is to learn embeddings of miRNAs and diseases from the network with remaining four subsets of associations. Then, the embeddings are combined with biological features about miRNAs and diseases to represent miRNA-disease pairs. Four subsets of associations (miRNA-disease pairs) are naturally used as positive instances. For the semi-supervised learning task, all other pairs (non-association) can be used as negative instances. Therefore, a RF-based prediction model is constructed. In the stage of prediction, the prediction model makes prediction for all non-association miRNA-disease pairs, which include the removed associations and real non-association pairs. Then, the prediction scores and their real labels about these pairs are used to calculate the metric scores. To avoid the bias of data split, we 
implement 10 runs of five-fold cross-validation for each model, and average performance is adopted.

We adopt several evaluation metrics: the area under the precise-recall curve (AUPR) and the area under the receiver-operating characteristic curve (AUC), F1-measure (F1), recall (REC) and precision (PRE), accuracy (ACC) and specificity (SPEC).

\section{Discussion of NEMII}

NEMII combines three types of feature vectors for describing miRNA-disease pairs, i.e. vectors based on miRNA-family associations, vectors based on disease semantic similarity and vectors based on Structural Deep Network Embedding (SDNE). Vectors based on Structural Deep Network Embedding (SDNE) are obtained from the known miRNA-disease bipartite network.

First, we try to discuss the influence of different feature combinations on the performance of NEMII. We consider different combinations of these three features: miRNA-family, disease similarity and SDNE feature by combining their feature vectors, and build the corresponding random forest-based prediction models. All models based on feature combinations are evaluated by 10 runs of five-fold cross-validation, and the results are shown in Table 1 . In general, combinations with SDNE feature produce better performance than combinations without SDNE feature. For example, the AUPR score of combination 2 (without SDNE feature) is around 57\% lower than that of the other four combinations (with SDNE feature). The AUC score of combination 2 (without SDNE feature) is $14 \%$ lower than that of other four combinations (with SDNE feature). Therefore, the results suggest that SDNE feature plays an important role in the prediction of miRNA-disease associations. Moreover, NEMII models which make use of SDNE feature, miRNA-family feature and disease similarity feature (combination 5) performs better than the models based on other combinations, indicating that the proposed method can well combine diverse features to achieve high-accuracy performances. To further demonstrate the advantage of NEMII, we conduct the statistical analysis to test the difference between NEMII and other feature combinations in terms of AUPR scores. The results show that although there is no significant difference between NEMII and the model based on combination 3 ( $p$-value $=0.3449$ by one-way ANOVA followed by post hoc Tukey test), and the model based on combination 4 ( $p$-value $=$ 0.2759 ), the NEMII model produces significantly better results than the model based on combination 1 $(p$-value $=0.001)$ and the model based on combination $2(p$-value $=0.001)$. The results demonstrate that NEMII which integrates SDNE feature, miRNA-family feature and disease similarity feature can produce good performance in the prediction of miRNA-disease associations.

The known miRNA-disease associations are important factors for predicting unobserved miRNA-disease associations. In order to test the influence of the number of known associations, i.e. data richness, we randomly remove 10, 20, 30\% known miRNA-disease associations from our dataset respectively, and then we perform 10 runs of five-fold cross-validation to evaluate NEMII on the datasets with fewer associations. As shown in Table 2, data richness greatly influenced the performance of our model, and AUPR and AUC scores decrease as more associations are removed. For example, the AUPR score is 0.6104 when there are no associations removed, but it decreases to 0.6001 when removing $10 \%$ associations. Then, the AUPR score decreases from 0.5956 (20\% associations removed) to 0.5863 ( $30 \%$ associations removed). The AUC score also decreases as associations are removed. More specifically, $10 \%$ decrease of associations can lead to around $0.1 \%$ decrease of the AUC score. Although the performances of NEMII decrease when reducing associations, NEMII still produces satisfying and robust results in the miRNA-disease predictions. The results demonstrate that SDNE is a robust embedding learning method, and can perform well even if the network becomes sparser.

Table 1 Performance of NEMII based on different feature combinations

\begin{tabular}{llllllll}
\hline & AUPR & AUC & F1 & ACC & REC & SPEC & PRE \\
\hline combination 1 & $0.6036 \pm 0.0018$ & $0.9252 \pm 0.0014$ & $0.6072 \pm 0.0020$ & $0.9955 \pm 0.0001$ & $0.4860 \pm 0.0052$ & $0.9992 \pm 0.0001$ & $0.8128 \pm 0.0158$ \\
combination 2 & $0.2630 \pm 0.0032$ & $0.7890 \pm 0.0056$ & $0.3338 \pm 0.0032$ & $0.9933 \pm 0.0000$ & $0.2360 \pm 0.0025$ & $0.9987 \pm 0.0000$ & $0.5681 \pm 0.0058$ \\
combination 3 & $0.6086 \pm 0.0015$ & $0.9284 \pm 0.0012$ & $0.6129 \pm 0.0031$ & $0.9956 \pm 0.0001$ & $0.4887 \pm 0.0069$ & $0.9992 \pm 0.0001$ & $0.8247 \pm 0.0160$ \\
combination 4 & $0.6085 \pm 0.0024$ & $0.9262 \pm 0.0018$ & $0.6115 \pm 0.0026$ & $0.9956 \pm 0.0000$ & $0.4836 \pm 0.0055$ & $0.9993 \pm 0.0001$ & $0.8366 \pm 0.0105$ \\
combination 5 & $0.6104 \pm 0.0012$ & $0.9293 \pm 0.0017$ & $0.6147 \pm 0.0025$ & $0.9956 \pm 0.0001$ & $0.4893 \pm 0.0060$ & $0.9993 \pm 0.0001$ & $0.8289 \pm 0.0164$ \\
\hline
\end{tabular}

* combination 1: SDNE feature alone

* combination 2: miRNA-family feature and disease similarity feature

* combination 3: SDNE feature and miRNA-family feature

* combination 4: SDNE feature and disease similarity feature

* combination 5: SDNE feature, miRNA-family feature and disease similarity feature 
Table 2 Performances of NEMII on datasets with fewer associations

\begin{tabular}{llllllll}
\hline Ratio & AUPR & AUC & F1 & ACC & REC & SPEC & PRE \\
\hline $0 \%$ & $0.6104 \pm 0.0012$ & $0.9293 \pm 0.0017$ & $0.6147 \pm 0.0025$ & $0.9956 \pm 0.0001$ & $0.4893 \pm 0.0060$ & $0.9993 \pm 0.0001$ & $0.8289 \pm 0.0164$ \\
$10 \%$ & $0.6001 \pm 0.0018$ & $0.9276 \pm 0.0011$ & $0.6045 \pm 0.0037$ & $0.9969 \pm 0.0001$ & $0.4811 \pm 0.0051$ & $0.9993 \pm 0.0001$ & $0.8176 \pm 0.0168$ \\
$20 \%$ & $0.5956 \pm 0.0030$ & $0.9266 \pm 0.0014$ & $0.6036 \pm 0.0040$ & $0.9965 \pm 0.0000$ & $0.4738 \pm 0.0091$ & $0.9995 \pm 0.0001$ & $0.8354 \pm 0.0169$ \\
$30 \%$ & $0.5863 \pm 0.0026$ & $0.9255 \pm 0.0010$ & $0.5946 \pm 0.0036$ & $0.9960 \pm 0.0001$ & $0.4620 \pm 0.0074$ & $0.9995 \pm 0.0001$ & $0.8390 \pm 0.0290$ \\
\hline
\end{tabular}

\section{Comparison with other network embeddings and other classifiers}

As discussed in Discussion of NEMII Section, features extracted by the embedding method SDNE are critical for building NEMII models. To demonstrate the advantage of SDNE, we also consider other popular network embedding methods: Laplacian Eigenmaps (LE) [23], High-Order Proximity preserved Embedding (HOPE) [24] and DeepWalk [18], and compare them with SDNE. LE keeps embeddings of two nodes close when these two nodes have high similarity. HOPE preserves high order proximity by decomposing the similarity matrix and using a generalized Singular Value Decomposition (SVD). DeepWalk uses random walks on graphs to learn latent representations of nodes and encodes them in a continuous space. These embedding methods usually have different parameters, and we set their parameters according to their publication and mainly discuss a common parameter: the dimension of node embeddings.

Here, we discuss the model performance under the different dimensions of node embeddings, ranging from 32 to $512\left(2^{k}, k=5,6,7,8,9\right)$. We respectively adopt these embedding methods to extract embedding features from the network, and then combine them with miRNA-family feature and disease similarity feature to build similar SDNE models. The results of all models are shown in Fig. 1. The y-axis denotes the AUPR and AUC scores obtained by the corresponding model, and the $\mathrm{x}$ axis denotes different dimensions of node embeddings. Clearly, the model using SDNE embedding leads to better AUPR scores and AUC scores than the models using other three embeddings over the different dimensions. To further demonstrate the advantage of SDNE, we conduct one-way ANOVA followed by post hoc Tukey test to test the difference between SDNE and other embedding methods in terms of AUPR scores. The results show that the SDNE model produces significantly better results than the HOPE model $(p$-value $=0.0027$ ) and the LAP model $(p$-value $=0.0019)$. The $p$-value between the SDNE model and the DeepWalk model is 0.0507, which suggests that there is no significant difference between the SDNE model and the DeepWalk model. Therefore, we conclude that SDNE method can learn more effective node embeddings in the miRNA-disease bipartite network and performs better than other three methods, because the miRNA-disease bipartite network is sparse and SDNE method was proved to be robust to sparse networks [25]. Anothers reason why SDNE works better than other embedding learning methods is that SDNE combines the autoencoder objective with the Laplacian eigenmaps objective.

Moreover, we observe from Fig. 1 (right) that the SDNE model using 128 dimensions produces the lowest AUC score of 0.9293 . In order to avoid overestimating
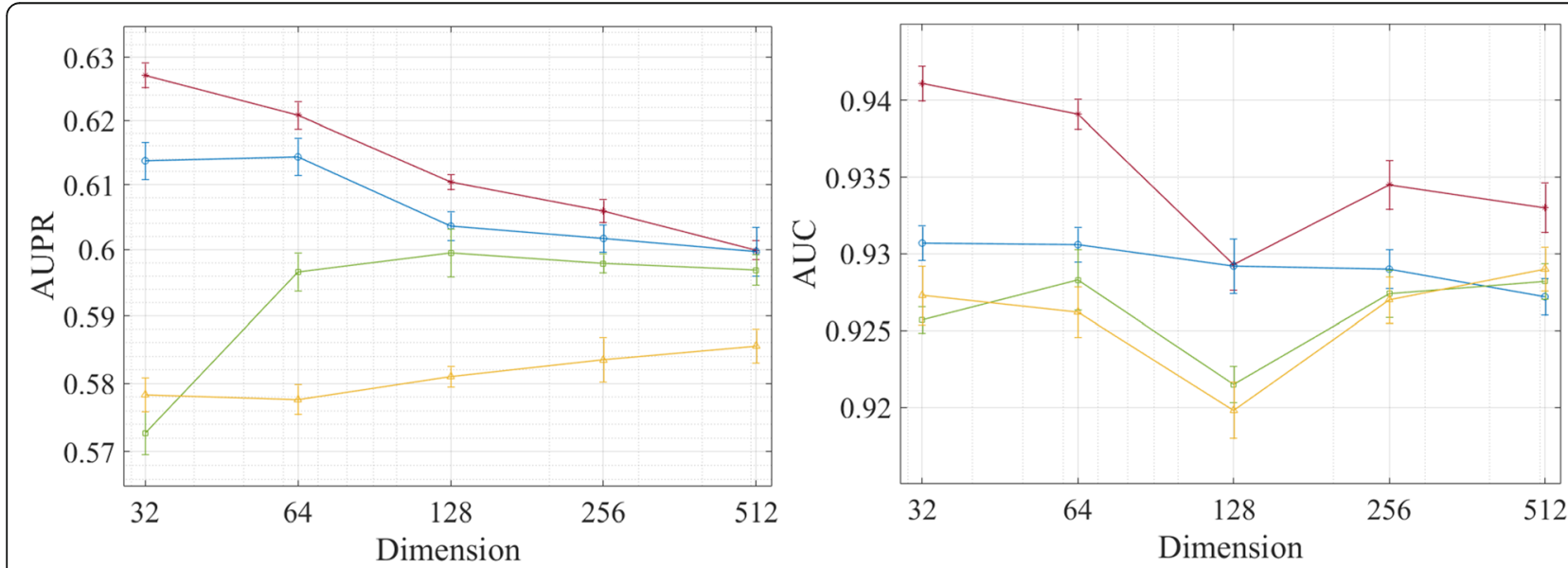

$\rightarrow$ SDNE $\rightarrow$ DeepWalk $\rightarrow$ HOPE $\stackrel{-}{- \text { LAP }}$

Fig. 1 AUPR and AUC of embedding methods based on different dimensions 
our model, we set the embedding vectors of SDNE as 128 dimensions in this study.

In order to show the advantage of random forest (RF) classifier, naive Bayes (NB), logistic regression (LR) and support vector machine (SVM) are used for comparison. By considering the number of trees ranging from 10 to 500 in a step of 10 , we set the number of trees in a RF classifier as 350 according to the performances of corresponding models. We adopt the radial basis function for SVM, and use the grid search to obtain optimal parameters $\mathrm{C}=1.0$ and gamma $=0.1$. The prediction models based on different classifiers are evaluated by five-fold cross-validation, and the results are shown in Table 3. Clearly, the model using RF classifier (AUC:0.9293, AUPR: 0.6104) performs better than the models using NB (AUC: 0.9103, AUPR: 0.1846), LR (AUC: 0.9023, AUPR: 0.2129) and SVM (AUC: 0.9021, AUPR: 0.0968). The results demonstrate that RF classifier is suitable for the miRNA-disease association prediction, because RF classifier is effective for the imbalanced and high-dimensional datasets.

We also implement weighted random forest to build the prediction model and compare it with the conventional random forest-based model. The conventional random forest assigns equal weights to output labels $(0,1)$ predicted by the decision trees of the random forest, while the weighted random forest assigns different weights to output labels $(0,1)$, denoted as $w_{i}=n_{-}$samples $/\left(2 * n_{i}\right)$, $i=\{0,1\}$, where $n_{-}$samples denotes the number of samples in the training set, and $n_{i}$ denotes the number of samples of each label $(0,1)$. The performance of the weighted random forest is shown in Table 3. Compared with the conventional RF, the weighted RF produces better AUC but lower AUPR and F1. In general, the weighted RF and conventional RF have the similar performances in the miRNA-disease association prediction, and thus conventional RF classifier is finally adopted in this work.

\section{Comparison with existing state-of-the-art methods}

To further demonstrate the advantages of NEMII, we compare it with three state-of-the-art methods: PBMDA [12], NTSMDA [11] and GRNMF [14], because they are latest methods with high-accuracy performances. PBMDA constructed a heterogeneous network based on miRNAmiRNA similarity, disease-disease similarity and known
miRNA-disease associations, and then scored miRNA-disease associations by using the number of paths from miRNAs to diseases. NTSMDA considered topological information of the miRNA-disease association network, and used the network-based resource allocation algorithm. GRNMF integrated disease semantic similarity and miRNA functional similarity, and used a graph regularized nonnegative matrix factorization framework to predict associations. Here, we implement these prediction methods according to their publications, and evaluate all models by using five-fold cross-validation under same conditions.

As shown in Table 4, NEMII produces the best performances, achieving the AUPR score of 0.6104, and the AUC score of 0.9293. PBMDA, NTSMDA and GRNMF produce the AUPR score of 0.2095, 0.0916 and 0.2446, and the AUC score of $0.9164,0.8857$ and 0.9128 respectively. The AUPR score of NEMII is significantly higher than the other three methods, and the AUC score is also higher than the other three methods. Moreover, we analyze the statistical differences between NEMII and the other three methods in terms of AUC scores, and we observe that there exists a very significant difference between NEMII and other three methods: PBMDA ( $p$ value $=0.001$ by one-way ANOVA followed by post hoc Tukey test), NTSMDA ( $p$-value $=0.001$ ) and GRNMF $(p$-value $=0.001)$. Therefore, NEMII produces significantly better results than PBMDA, NTSMDA and GRNMF in the cross-validation experiment.

Further, we compare the predictive performances of four methods for specified diseases. We select three diseases of wide interests: "breast neoplasms", "lung neoplasms" and "prostatic neoplasms", and then we explore the results of different methods on these three diseases. Breast neoplasm develops from breast tissues which are highly prevalent in women. Lung neoplasm is a kind of malignant lung neoplasm caused by uncontrolled growth of lung tissue cells. Prostatic neoplasm is a kind of malignant neoplasm occurring in the prostate. We implement 10 runs of five-fold cross-validation and then obtain the prediction results of all these methods for each disease. As shown in Fig. 2 (left), NEMII produces significantly higher AUPR scores than PBMDA, NTSMDA and GRNMF for all three diseases. For example, the AUPR score for breast neoplasm produced by NEMII is 0.8476 , which is $37.78 \%$ higher than the AUPR

Table 3 Performance of models based on different classifiers

\begin{tabular}{llllllll}
\hline Classifiers & AUPR & AUC & F1 & ACC & REC & SPEC & PRE \\
\hline RF & $0.6104 \pm 0.0012$ & $0.9293 \pm 0.0017$ & $0.6147 \pm 0.0025$ & $0.9956 \pm 0.0001$ & $0.4893 \pm 0.006$ & $0.9993 \pm 0.0001$ & $0.8289 \pm 0.0164$ \\
NB & $0.1846 \pm 0.0008$ & $0.9103 \pm 0.0089$ & $0.2528 \pm 0.0028$ & $0.9892 \pm 0.0004$ & $0.2572 \pm 0.0124$ & $0.9944 \pm 0.0005$ & $0.2532 \pm 0.0056$ \\
LR & $0.2129 \pm 0.0008$ & $0.9023 \pm 0.0008$ & $0.2734 \pm 0.0017$ & $0.9884 \pm 0.0004$ & $0.3078 \pm 0.0094$ & $0.9933 \pm 0.0005$ & $0.2480 \pm 0.0096$ \\
SVM & $0.0968 \pm 0.0034$ & $0.9021 \pm 0.0010$ & $0.1718 \pm 0.0036$ & $0.9740 \pm 0.0012$ & $0.3761 \pm 0.0144$ & $0.9783 \pm 0.0013$ & $0.1121 \pm 0.0037$ \\
weighted RF & $0.5944 \pm 0.0014$ & $0.9336 \pm 0.0014$ & $0.5920 \pm 0.0025$ & $0.9953 \pm 0.0001$ & $0.4741 \pm 0.0085$ & $0.9991 \pm 0.0001$ & $0.7913 \pm 0.0233$ \\
\hline
\end{tabular}


Table 4 Performances of NEMII, PBMDA, NTSMDA and GRNMF

\begin{tabular}{llllllll}
\hline Methods & AUPR & AUC & F1 & ACC & REC & SPEC & PRE \\
\hline NEMII & $0.6104 \pm 0.0012$ & $0.9293 \pm 0.0017$ & $0.6147 \pm 0.0025$ & $0.9956 \pm 0.0001$ & $0.4893 \pm 0.0060$ & $0.9993 \pm 0.0001$ & $0.8289 \pm 0.0164$ \\
PBMDA & $0.2095 \pm 0.0015$ & $0.9164 \pm 0.0005$ & $0.2676 \pm 0.0021$ & $0.9892 \pm 0.0005$ & $0.2759 \pm 0.0139$ & $0.9944 \pm 0.0006$ & $0.2642 \pm 0.0103$ \\
NTSMDA & $0.0916 \pm 0.0012$ & $0.8857 \pm 0.0009$ & $0.1410 \pm 0.0013$ & $0.9740 \pm 0.0015$ & $0.2988 \pm 0.0171$ & $0.9788 \pm 0.0017$ & $0.0931 \pm 0.0020$ \\
GRNMF & $0.2446 \pm 0.0024$ & $0.9128 \pm 0.0008$ & $0.3192 \pm 0.0137$ & $0.9945 \pm 0.0005$ & $0.2989 \pm 0.0127$ & $0.9897 \pm 0.0004$ & $0.3066 \pm 0.0016$ \\
\hline
\end{tabular}

score of 0.5274 produced by PBMDA (the highest among PBMDA, NTSMDA, and GRNMF). And as shown in Fig. 2 (right), NEMII also produces higher AUC scores than PBMDA, NTSMDA and GRNMF for all three diseases. For example, the AUC score of prostatic neoplasm obtained by NEMII is 0.9296, which is 9.76\% higher than 0.8389 obtained by GRNMF (the highest among PBMDA, NTSMDA, and GRNMF). Therefore, we can conclude that NEMII outperforms PBMDA, NTSMDA and GRNMF in predicting miRNAs for three specified diseases.

We further investigate what the percentage of removed known miRNA-disease associations could be covered by NEMII and other approaches. We randomly remove 10, 20 and 30\% known miRNA-disease associations respectively, then train models based on the remained known and unknown associations and test what percentage of removed associations could be recovered. We use REC values to represent the results. The performances of NEMII and other approaches are shown in Fig. 3. Clearly, NEMII leads to better REC values than other approaches when removing 10, 20 and 30\% known associations. To further demonstrate the advantage of NEMII, we conduct one-way ANOVA followed by post hoc Tukey test to test the difference between NEMII and other approaches in terms of REC values. The results show that NEMII produces significantly better results than PBMDA ( $p$-value $=0.001)$, NTSMDA ( $p$ value $=0.001)$ and GRNMF $(p$-value $=0.001)$. Therefore, we can conclude that NEMII outperforms PBMDA, NTSMDA and GRNMF in recovering the removed known miRNA-disease associations.

\section{Case studies}

Here, we use case studies to test the capability of our method for predicting unknown miRNA-disease associations. We build the NEMII model by using all miRNAdisease associations in our dataset, and make predictions for non-association miRNA-disease pairs. Since all known associations in HMDD database are used to build models, the predicted associations have to be verified by public literature and other available sources. We list top 10 miRNA-disease associations predicted by NEMII in Table 5, and found evidence to confirm 5 out of them. For example, hsa-let-7c expression was found to be related to non-atrophic gastritis and atrophic gastritis [26]. Hsa-mir-103a-2 expression was downregulated in patients with myelodysplastic syndromes [27]. The expression of hsa-let-7e has an influence of time-dependent suppression on Biliary Atresia [28]. Increased expression of hsa-mir-1179 can inhibit breast cancer cell metastasis by modulating Notch signaling pathway [29]. The expression of hsa-mir-1179 was found to be associated with Hepatocellular Carcinoma. Therefore, our method

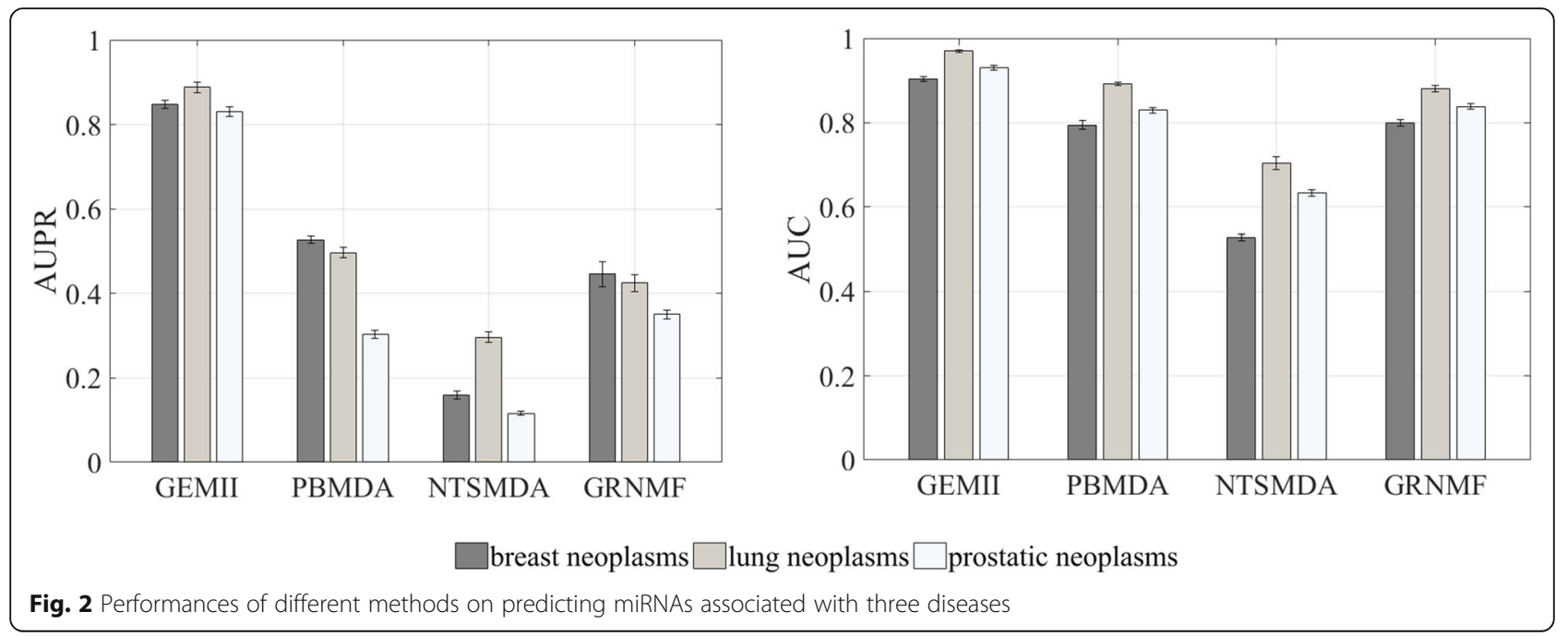




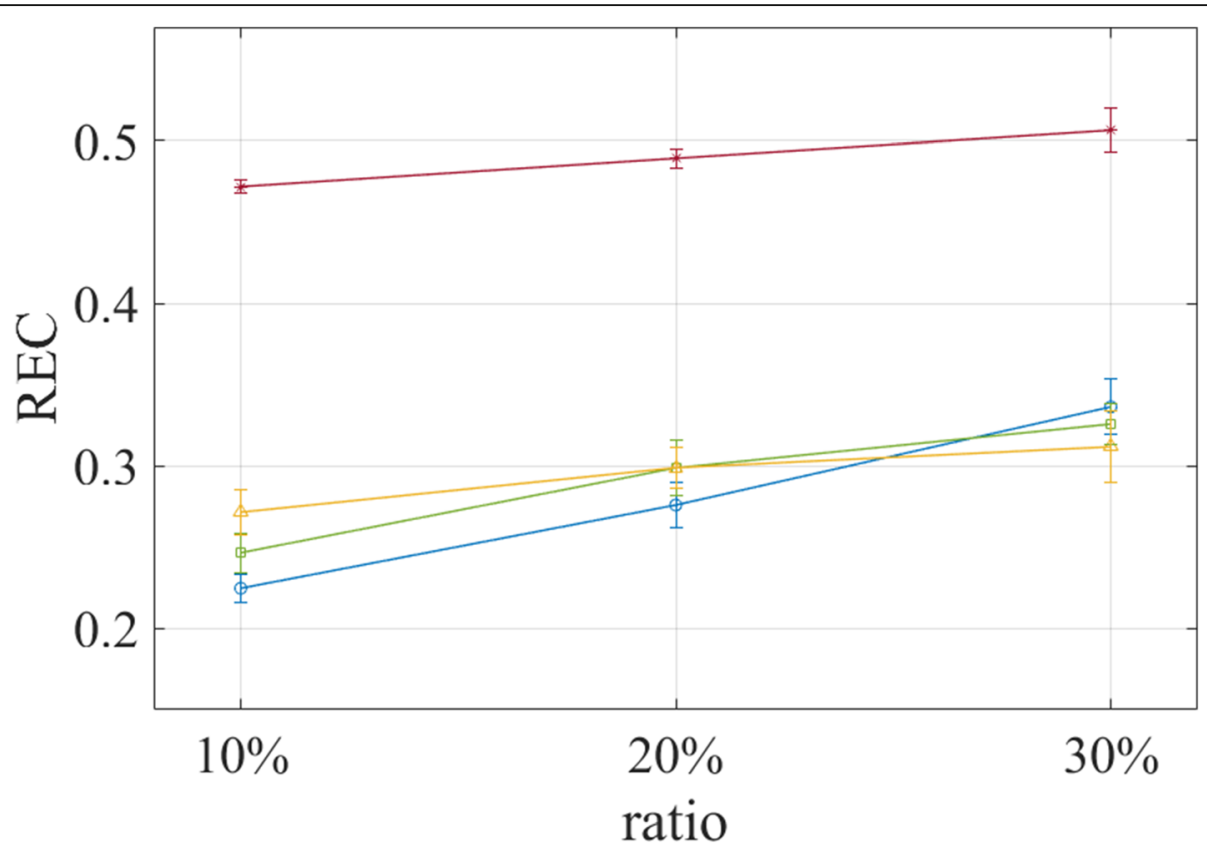

$\rightarrow$ NEMII $\rightarrow$ PBMDA - NTSMDA $\backsim$ GRNMF

Fig. 3 Performances of recovering associations of NEMII and other approaches

can help to identify unknown miRNA-disease associations.

Moreover, we predict miRNAs which are associated with three diseases "breast neoplasms", "lung neoplasms" and "prostatic neoplasms" mentioned in Comparison with existing state-of-the-art methods Section. Then we select the top 10 miRNAs associated with each disease, and try to obtain evidence to confirm our findings. As shown in Table 6, we have evidence to support $5 \mathrm{miR}$ NAs associated with breast neoplasms. For example, miR-1179 expression was found to be frequently downregulated in breast cancer tissues and cell lines [29]. We find evidences to confirm that 5 miRNAs are associated with lung neoplasms. For example, hsa-mir-376c can suppress non-small-cell lung cancer cell growth and invasion by targeting LRH-1-mediated Wnt signaling pathway [35]. Moreover, 4 miRNAs are found to be associated with prostatic neoplasms. For example, hsamir-1179 was found to be one of the most highly upregulated miRNAs from the observation of micro dissected prostate tumor cells [39], and hsa-mir-10a was found to be one of the most highly expressed miRNAs in prostate tumors [39]. These evidence shows that these disease-related miRNAs have close relationships with breast neoplasms, lung neoplasms and prostatic neoplasms and may be of potential use in the diagnosis of these diseases. Therefore, NEMII is useful for predicting miRNAs associated with given diseases.

Table 5 The top 10 miRNA-disease associations predicted by our method

\begin{tabular}{llll}
\hline miRNA & Disease & Rank & Evidence \\
\hline hsa-let-7c & Crohn Disease & 1 & N.A. \\
hsa-let-7c & Gastritis, Atrophic & 2 & {$[26]$} \\
hsa-let-7e & Lymphoproliferative Disorders & 3 & N.A. \\
hsa-let-7e & Giant Cell Tumors & 4 & N.A. \\
hsa-mir-103a-2 & Myelodysplastic Syndromes & 5 & {$[27]$} \\
hsa-let-7e & Biliary Atresia & 6 & {$[28]$} \\
hsa-mir-10a & Carotid Artery Diseases & 7 & N.A. \\
hsa-mir-10b & Eczema & 8 & N.A. \\
hsa-mir-1179 & Breast Neoplasms & 9 & {$[29]$} \\
hsa-mir-1179 & Carcinoma, Hepatocellular & 10 & https://figshare.com/articles/Liver_hepatocellular_carcinoma/6804233 \\
\hline
\end{tabular}


Table 6 Predicted miRNAs associated with three diseases

\begin{tabular}{|c|c|c|c|}
\hline Disease & miRNA & Rank & Evidence \\
\hline \multirow[t]{10}{*}{ breast neoplasms } & hsa-mir-1179 & 1 & [29] \\
\hline & hsa-mir-1180 & 2 & {$[30]$} \\
\hline & hsa-mir-106a & 3 & [31] \\
\hline & hsa-mir-377 & 4 & N.A. \\
\hline & hsa-mir-1909 & 5 & N.A. \\
\hline & hsa-mir-181c & 6 & N.A. \\
\hline & hsa-mir-1202 & 7 & N.A. \\
\hline & hsa-mir-1296 & 8 & {$[32]$} \\
\hline & hsa-mir-2110 & 9 & N.A. \\
\hline & hsa-mir-711 & 10 & [33] \\
\hline \multirow[t]{10}{*}{ lung neoplasms } & hsa-mir-1180 & 1 & N.A. \\
\hline & hsa-mir-1179 & 2 & [34] \\
\hline & hsa-mir-376c & 3 & {$[35]$} \\
\hline & hsa-mir-500b & 4 & N.A. \\
\hline & hsa-mir-1293 & 5 & {$[36]$} \\
\hline & hsa-mir-296 & 6 & {$[37]$} \\
\hline & hsa-mir-1183 & 7 & N.A. \\
\hline & hsa-mir-99b & 8 & {$[38]$} \\
\hline & hsa-mir-298 & 9 & N.A. \\
\hline & hsa-mir-2110 & 10 & N.A. \\
\hline \multirow[t]{10}{*}{ prostatic neoplasms } & hsa-mir-103a-2 & 1 & N.A. \\
\hline & hsa-mir-1179 & 2 & [39] \\
\hline & hsa-mir-10b & 3 & N.A. \\
\hline & hsa-mir-10a & 4 & [39] \\
\hline & hsa-mir-1180 & 5 & {$[40]$} \\
\hline & hsa-mir-147a & 6 & N.A. \\
\hline & hsa-mir-217 & 7 & N.A. \\
\hline & hsa-mir-125a & 8 & [41] \\
\hline & hsa-mir-624 & 9 & N.A. \\
\hline & hsa-mir-630 & 10 & N.A. \\
\hline
\end{tabular}

\section{Conclusion}

The identification of miRNA-disease associations plays an important role in furthering understanding the molecular mechanism of many human diseases. In this work, we propose a novel computational method, called NEMII, to predict unknown miRNA-disease associations. Different from existing methods which mainly make use of biological features of miRNAs and diseases, NEMII extracts the embedding representations of miRNAs and diseases from the miRNA-disease bipartite network, and further combines them with biological features to build the prediction model. Experimental results reveal that NEMII performs better than the models using biological features alone and models using embedding representations alone, and SDNE produces better results than using other network embedding methods.
NEMII also produces better results when compared with other state-of-the-art methods. Case studies show that NEMII can predict novel miRNA-disease associations, and can predict miRNAs associated with given diseases. In conclusion, NEMII is a promising method for the miRNA-disease association prediction.

\section{Methods \\ Datasets}

There are several databases about miRNA-disease associations, e.g. the human microRNA disease database (HMDD) [42], the database of differentially expressed miRNAs in human cancers (dbDEMC) [43] and the database for microRNA deregulation in human disease (miR2Disease) [44]. The databases lay the basis for developing computational methods to predict unobserved miRNAdisease associations.

In this study, we compile our datasets from HMDD database v2.0, miRBase and Medical Subject Heading (MeSH). HMDD [45] is a database which contains human miRNA-disease associations and comprehensive annotations. We downloaded experimentally confirmed miRNA-disease associations from HMDD, including 578 miRNAs, 383 diseases and 6448 associations. The database miRBase [46] is an online repository of miRNA sequences and the experimental miRNA-family relationships. We collected miRNA-family associations from miRBase, including 17,613 miRNAs and 1983 families; a miRNA belongs to a family and a family contains more than one miRNA. MeSH is a comprehensive medical vocabulary, which is useful for exploring the relationship between different diseases. We downloaded disease descriptors from MeSH. The relationships of diseases can be transformed into a directed acyclic graph (DAG), and the nodes of a DAG represent the diseases and the edges represent the relationships of different diseases. DAGs can be used to calculate disease semantic similarity [12].

We removed miRNAs without family information as well as diseases without MeSH descriptors. Finally, we obtained 4479 miRNA-disease associations between 412 miRNAs and 314 diseases, 278 miRNA-family associations between 412 miRNAs and 278 families, and $\mathrm{MeSH}$ descriptors for 314 diseases.

\section{Pipeline of network embedding-based multiple information integration method}

For the following study, we first introduce several mathematical notations. Given miRNAs $M=\left\{M_{1}, M_{2}, \ldots, M_{m}\right\}$, diseases $D=\left\{D_{1}, D_{2}, \cdots, D_{n}\right\}$ and miRNA-disease associations, our task is to predict unknown miRNA-disease associations based on known associations and biological features. The associations between $m$ miRNAs and $n$ diseases can be represented by a binary matrix $A$, in which each row represents a miRNA and each column 
represents a disease. If the $i$ th miRNA is associated with the $j$ th disease, $A_{i j}=1$; otherwise, $A_{i j}=0$. Formally, $m$ miRNAs, $n$ diseases and their known associations can be formulated as a network, in which miRNAs and diseases are taken as nodes and their associations are taken as edges. The network can be represented by a $(m+n) \times(m+n)$ adjacency matrix $G$, defined as $G=\left[\begin{array}{cc}0 & A \\ A^{T} & 0\end{array}\right]$.

The studies [47-53] have revealed that combining diverse information helps to improve the accuracy of prediction models in bioinformatics. The network embedding-based multiple information integration method (NEMII) is to combine biological features of miRNAs and diseases with their embedding representations. As described in Fig. 4, NEMII takes several steps to construct a prediction model. First, miRNAfamily associations are used to represent miRNAs; $\mathrm{MeSH}$ information of diseases are used to calculate disease-disease similarity and then represent diseases. Second, the known miRNA-disease associations are formulated as a bipartite network, and node embeddings in the bipartite network are learned by using SDNE and then used to represent miRNAs and diseases. Third, all representations of miRNAs and diseases are combined to represent miRNA-disease pairs.
Finally, a prediction model is constructed based on the miRNA-disease pairs by using random forest.

\section{Constructing feature vectors to represent miRNA-disease pairs}

In order to predict miRNA-disease associations, we should use a reasonable way to represent features of miRNA-disease pairs. To our best knowledge, most existing methods heavily rely on biological features of miRNAs and diseases, such as miRNA-family association, miRNA-functional similarity and disease semantic similarity. Besides, the features learned from the miRNA-disease association network can be taken into account. Features from known miRNA-disease bipartite network are seldom considered, but they are effective for preserving the property of the network. Therefore, there are three types of feature vectors for describing miRNAdisease pairs, i.e. vectors based on miRNA-family associations, vectors based on disease semantic similarity and vectors based on Structural Deep Network Embedding.

\section{Representing miRNAs with miRNA-family associations}

There is an assumption that miRNAs in the same family may perform similar biological functions. Here, we utilize miRNA-family associations to represent miRNA

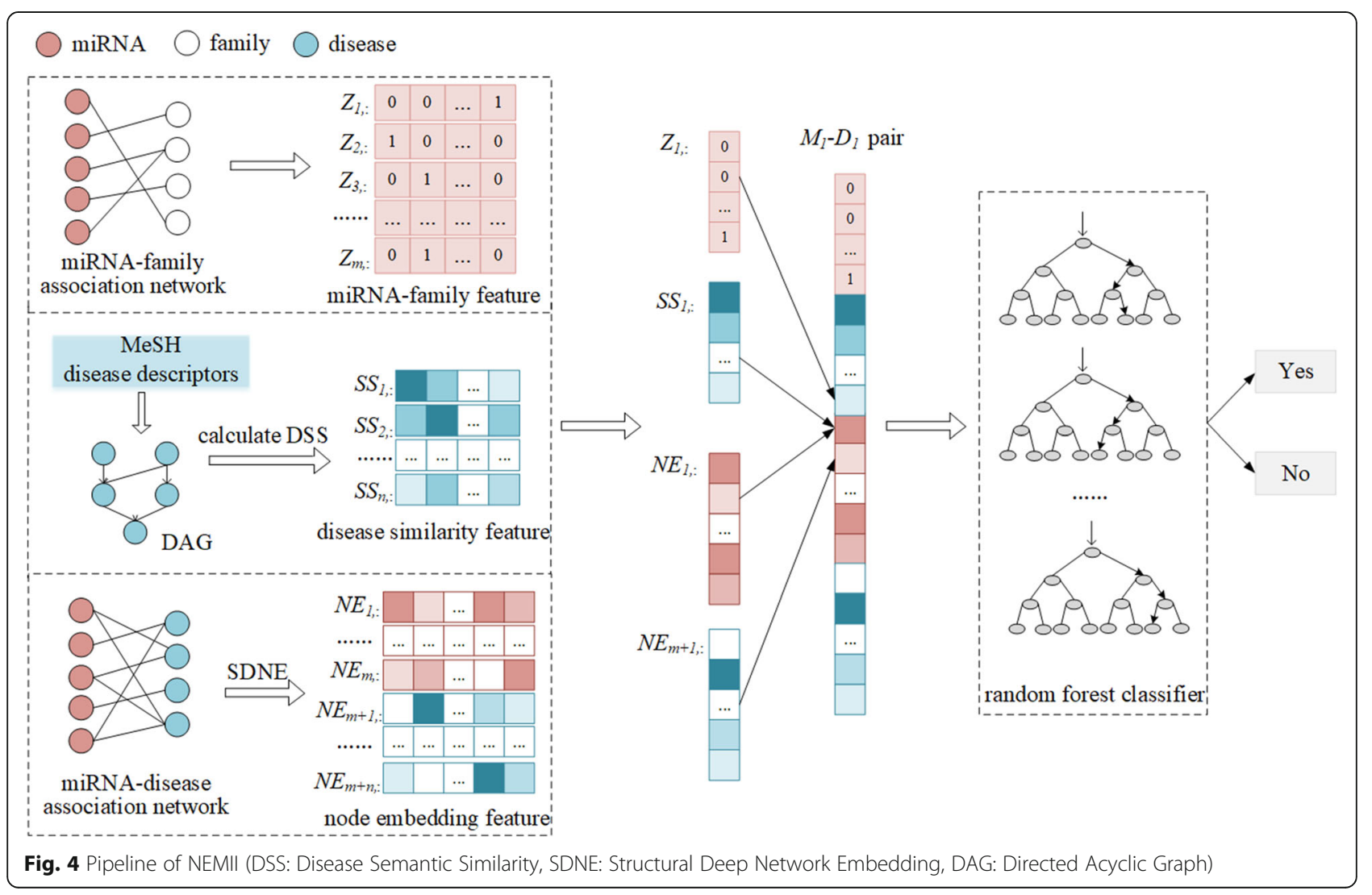


biological feature. For miRNAs $M=\left\{M_{1}, M_{2}, \ldots, M_{m}\right\}$, families $F=\left\{F_{1}, F_{2}, \cdots, F_{t}\right\}$, and their associations, we can formulate them as a bipartite network, which uses miRNAs and families as nodes and uses their associations as edges. The bipartite network can be represented by a $m \times t$ adjacency matrix $Z$. This is, $Z_{i j}=1$ if miRNA $M_{i}$ belongs to family $F_{j}$; otherwise, $Z_{i j}=0$. Then, for a specific miRNA $M_{i}$, we use the $i$ th row vector of $Z$, namely $Z_{i, \text { : }}$ to denote its biological feature.

\section{Representing diseases with disease semantic similarity}

Inspired by previous works [12], diseases and their relationships can be transformed into a directed acyclic graph (DAG), and DAGs can be used to calculate disease semantic similarity.

For a given disease $D$, the directed acyclic graph $D A G_{D}=\left(V_{D}, E_{D}\right) . V_{D}$ denotes the node set including $D$ and other diseases which have relationships with $D$, and $E_{D}$ denotes the edge set which contains the links from parent disease to child disease. According to DAG, the semantic contribution of disease $d$ in $D A G_{D}$ to disease $D$ can be denoted as:

$$
S_{\mathrm{D}}(d)=\left\{\begin{array}{c}
1 \text { if } d=D \\
\max \left\{\Delta_{*} * S_{D}\left(d^{\prime}\right) \mid d^{\prime} \in \text { children of } d\right\} \text { if } d \neq D
\end{array}\right.
$$

Here, we set $\Delta_{*}=0.5$. The semantic value of disease $D$ can be calculated as:

$$
S V_{D}=\sum_{d \in V_{D}} S_{D}(d)
$$

The semantic similarity between disease $D_{i}$ and disease $D_{j}$ is calculated by:

$$
S S\left(D_{i}, D_{j}\right)=\frac{\sum_{d \epsilon V_{D_{i}} \cap V_{D_{j}}}\left(S_{D_{i}}(d)+S_{D_{j}}(d)\right)}{S V_{D_{i}}+S V_{D_{j}}}
$$

where $S_{D_{i}}(d)$ is the semantic contribution of $d$ to disease $D_{i}$, and $S V_{D_{i}}$ is the semantic value of $D_{i}$; for $D_{j}$, the meanings of $S_{D_{j}}(d)$ and $S V_{D_{j}}$ are similar to $D_{i}$. Then, the semantic similarity between all the diseases can be represented as a $n \times n$ matrix $S S$, and the value in row $i$ and column $j$ of $S S$ represents the disease semantic similarity between $D_{i}$ and $D_{j}$. For a specific disease $D_{i}$, we

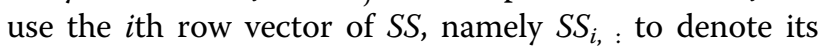
biological feature.

\section{Representing miRNA-disease pairs with structural deep network embedding}

Recently, the network embedding methods show the great potentials of analyzing networks, especially extracting node features. Compared with traditional network analysis methods, which calculate network density, degree statistics and clustering coefficient, the network embedding methods generate low-dimensional vectors that reflect the comprehensive characteristic of networks. Since known miRNA-disease associations could be formulated as a miRNA-disease association network, we naturally use the network embedding methods to extract the features from it. We consider several popular network embedding methods in this work, and compare them in the Comparison with other network Embeddings and other classifiers Section. Because the Structural Deep Network Embedding method performs best among all embedding methods, it is finally adopted for the miRNA-disease association prediction.

Structural Deep Network Embedding method, namely SDNE, is semi-supervised deep model, which has multiple layers of non-linear functions to capture the highly non-linear network structure through firstorder and second-order proximity. Since SDNE jointly optimizes first-order and second-order proximity, SDNE is robust to sparse networks [54], and outperforms popular network embedding methods in many applications, i.e. graph reconstruction, link prediction and visualization [55].

Given a network with $N$ nodes and the adjacency matrix $G=\left(G_{i j}\right)$, we introduce how to learn the node embedding representations. SDNE utilizes the traditional deep autoencoder [56], which has two components: encoder and decoder. The encoder consists of multiple non-linear functions that maps initial representation of each node $x_{i}$ to a low-rank space through $K$ hidden layers, and the low-rank vector is denoted as $y_{i} . x_{i}=G_{i}$, ,where $G_{i, \text { : }}$ is the $i$ th row of the adjacency matrix $G$ mentioned in Pipeline of network embedding-based multiple information integration method Section. The decoder attempts to reconstruct the representation of the node, and the reconstructed vector is denoted by $\hat{x}_{i}$.

The first-order proximity is used as the supervised information to preserve the local network structure, and its objective function is as follows:

$$
L_{1 s t}=\sum_{i, j=1}^{N} G_{i j}\left\|\left(y_{i}-y_{j}\right)\right\|_{2}^{2}
$$

where $y_{i}$ is the low-rank representation of node $i$, and $y_{j}$ is the low-rank representation of node $j$.

The second-order proximity is used as the unsupervised information to capture the global network structure, and its objective function is as follows:

$$
L_{2 n d}=\|(\hat{X}-X) \odot B\|_{F}^{2}
$$

where $\odot$ means the Hadamard product. $B$ is a $N \times N$ matrix. $B_{i j}=1$, if $G_{i j}=0$, else $b_{i j}=\beta$, where $\beta$ is free parameter and $\beta>1 . X=\left[x_{1}, x_{2}, \cdots, x_{N}\right]^{T}, \hat{X}=\left[\hat{x}_{1}, \hat{x}_{2}, \cdots, \hat{x}_{N}\right]^{T}$. 
Moreover, a $L 2$-norm regularization term is used to prevent overfitting and defined as follows:

$$
L_{\text {reg }}=\frac{1}{2} \sum_{k=1}^{K}\left(\left\|W^{(k)}\right\|_{F}^{2}+\left\|\hat{W}^{(k)}\right\|_{F}^{2}\right)
$$

where $K$ is the number of hidden layers, $W^{k}$ and $\hat{W}^{(k)}$ are the $k$ th-layer weight matrices.

SDNE combines Eqs. 1, 2 and 3, and minimizes the following objective function:

$$
\begin{aligned}
L_{\text {mix }} & =L_{2 n d}+\alpha L_{1 s t}+v L_{\text {reg }} \\
& =\|(\hat{X}-X) \odot B\|_{F}^{2}+\alpha \sum_{i, j=1}^{N} G_{i i j}\left\|\left(y_{i}-y_{j}\right)\right\|_{2}^{2} \\
& +v \frac{1}{2} \sum_{k=1}^{K}\left(\left\|W^{(k)}\right\|_{F}^{2}+\left\|\hat{W}^{(k)}\right\|_{F}^{2}\right)
\end{aligned}
$$

More details about SDNE are available in [54].

We can apply SDNE to the miRNA-disease bipartite network with the adjacency matrix $G$, and obtain a $(m+n) \times d$ embedding matrix $N E$, where $d$ is a free parameter that denotes the dimension of node embeddings. $m$ and $n$ are mentioned in Pipeline of network embedding-based multiple information integration method Section. The rows of $N E$, namely $N E_{i}$, : correspond to the embeddings of $m$ miRNA node and $n$ disease nodes.

We also consider other popular network embedding methods Laplacian Eigenmaps (LE) [23], High-Order Proximity preserved Embedding (HOPE) [24] and DeepWalk [18], and compare SDNE with them in Comparison with other network Embeddings and other classifiers Section.

\section{Model construction}

We combine three types of features to describe miRNA-disease pairs, and then use them to build classification-based models. Specifically, four feature vectors: miRNA-family feature vectors, disease semantic similarity feature vectors, miRNA embedding feature vectors and disease embedding feature vectors are merged. We adopt random forest as the classification engine to classify miRNA-disease pairs. Random forest is an ensemble learning method containing multiple classification trees [57]. Each tree is constructed by using a bootstrap sample of the training dataset. For each node within each tree, a randomly selected subset of the input features is used. Then the classification output of random forest is determined by the majority classification of all the trees. Random forest is well-known for its ability to deal with unbalanced datasets [58], and studies also demonstrated that random forest has good performances for bioinformatics problems [22, 59].
To the best of our knowledge, there are a great number of popular classifiers in bioinformatics, such as logistic regression, naive Bayes and support vector machine. We also compare random forest with these classifiers in Comparison with other network Embeddings and other classifiers Section.

\section{Additional file}

Additional file 1: Figure S1. Five-fold cross-validation (CV) for Network Embedding-based Multiple Information Integration Method. (PDF 312 kb)

\begin{abstract}
Abbreviations
AUC: Area under the receiver-operating characteristic curve; AUPR: Area under the precision-recall curve; DAG: Directed acyclic graph; dbDEMC: Database of differentially expressed miRNAs in human cancers; DSS: Disease semantic similarity; HMDD: Human microRNA disease database; HOPE: High-Order Proximity preserved Embedding; LE: Laplacian Eigenmaps; LR: Logistic regression; MeSH: Medical subject heading; miR2Disease: Database for microRNA deregulation in human disease; NB: Naive Bayes; NEMII: Network Embedding-based Multiple Information Integration method; RF: Random forest; SDNE: Structural Deep Network Embedding; SVD: Singular Value Decomposition; SVM: Support vector machine
\end{abstract}

\section{Acknowledgements}

Not applicable.

\section{Authors' contributions}

WZ conceived the project; $Y G$ and WZ designed the experiments; YG performed the experiments; YG, YN, WZ and XL wrote the paper. All authors read and approved the final manuscript.

\section{Funding}

This work is supported by the National Natural Science Foundation of China (61772381, 61572368), the Fundamental Research Funds for the Central Universities (2042017kf0219, 2042018kf0249), National Key Research and Development Program (2018YFC0407904), Huazhong Agricultural University Scientific \& Technological Self-innovation Foundation. The funders have no role in study design, data collection, data analysis, data interpretation, or writing of the manuscript.

\section{Availability of data and materials}

The datasets generated and/or analysed during this study are available under open licenses in the data repository, https://github.com/ BioMedicalBigDataMiningLab/NEMII.

\section{Ethics approval and consent to participate} Not applicable.

\section{Consent for publication}

Not applicable.

\section{Competing interests}

The authors declare that they have no competing interests.

\section{Author details}

${ }^{1}$ School of Computer Science, Wuhan University, Wuhan 430072, China. ${ }^{2}$ School of Mathematics and Statistics, South-Central University for Nationalities, Wuhan 430074, China. ${ }^{3}$ College of Informatics, Huazhong Agricultural University, Wuhan 430070, China. 


\section{Received: 17 May 2019 Accepted: 29 August 2019} Published online: 12 September 2019

\section{References}

1. Ribeiro AO, Schoof CR, Izzotti A, Pereira LV, Vasques LR. MicroRNAs: modulators of cell identity, and their applications in tissue engineering. Microrna. 2014;3(1):45-53.

2. Vasudevan S, Tong $Y$, Steitz JA. Switching from repression to activation: MicroRNAs can up-regulate translation. Science. 2007;318(5858):1931-4.

3. Xantha K, Victor A. Developmental biology. Encountering microRNAs in cell fate signaling. Science. 2005;310(5752):1288.

4. Miska EA. How microRNAs control cell division, differentiation and death. Curr Opin Genet Dev. 2005;15(5):563-8.

5. Cheng AM, Byrom MW, Shelton J, Ford LP. Antisense inhibition of human miRNAs and indications for an involvement of miRNA in cell growth and apoptosis. Nucleic Acids Res. 2005;33(4):1290-7.

6. Xu P, Guo M, Hay BA. MicroRNAs and the regulation of cell death. Trends Genet. 2004;20(12):617-24.

7. Ming L, Qipeng Z, Min D, Jing M, Yanhong G, Wei G, Qinghua C. An analysis of human microRNA and disease associations. PLoS One. 2008;3(10):e3420.

8. Iorio MV, Manuela F, Chang-Gong L, Angelo V, Riccardo S, Silvia S, Eros M, Massimo P, Muller F, Manuela C. MicroRNA gene expression deregulation in human breast cancer. Cancer Res. 2005;65(16):7065.

9. Latronico MV, Catalucci D, Condorelli G. Emerging role of microRNAs in cardiovascular biology. Circ Res. 2007;101(12):1225-36.

10. Lynam-Lennon N, Maher SG, Reynolds JV. The roles of microRNA in cancer and apoptosis. Biol Rev Camb Philos Soc. 2010;84(1):55-71.

11. Sun D, Li A, Feng H, Wang M. NTSMDA: prediction of miRNA-disease associations by integrating network topological similarity. Mol BioSyst. 2016;12(7):2224.

12. You ZH, Huang ZA, Zhu Z, Yan GY, Li ZW, Wen Z, Chen X. PBMDA: a novel and effective path-based computational model for miRNA-disease association prediction. PLoS Comput Biol. 2017;13(3):e1005455.

13. Chen X, Wu QF, Yan GY. RKNNMDA: ranking-based KNN for MiRNA-disease association prediction. RNA Biol. 2017;14(7):1.

14. Xiao Q, Luo J, Liang C, Cai J, Ding P. A graph regularized non-negative matrix factorization method for identifying microRNA-disease associations. Bioinformatics. 2017;34(2):239-48.

15. Chen X, Wang L, Qu J, Guan NN, Li JQ. Predicting miRNA-disease association based on inductive matrix completion. Bioinformatics. 2018; 34(24):4256-65.

16. LuO J, Ding P, Liang C, Chen X. Semi-supervised prediction of human miRNA-disease association based on graph regularization framework in heterogeneous networks. Neurocomputing. 2018;294:29-38.

17. Chen X, Xie D, Wang L, Zhao Q, You ZH, Liu H. BNPMDA: bipartite network projection for MiRNA-disease association prediction. Bioinformatics. 2018,34(18):3178-3186.

18. Perozzi B, Al-Rfou R, Skiena S. DeepWalk: Online Learning of Social Representations; 2014. p. 701-10.

19. Aditya Grover JL: node2vec: Scalable Feature Learning for Networks. In: Acm Sigkdd International Conference on Knowledge Discovery \& Data Mining; 2016. p. 855-864.

20. Zong N, Kim H, Ngo V, Harismendy O. Deep mining heterogeneous networks of biomedical linked data to predict novel drug-target associations. Bioinformatics. 2017;33(15):2337-2344.

21. Li G, Luo J, Xiao Q, Liang C, Ding P, Cao B. Predicting MicroRNA-disease associations using network topological similarity based on DeepWalk. IEEE Access. 2017;5:24032-24039.

22. Liu X, Yang Z, Sang S, Zhou Z, Wang L, Zhang Y, Lin H, Wang J, Xu B. Identifying protein complexes based on node embeddings obtained from protein-protein interaction networks. Bmc Bioinformatics. 2018;19(1):332.

23. Belkin M, Niyogi P. Laplacian Eigenmaps for dimensionality reduction and data representation. Neural Computation. 2003;15(6):1373-1396.

24. Ou M, Cui P, Pei J, Zhang Z, Zhu W. Asymmetric transitivity preserving graph embedding. In: The ACM SIGKDD International Conference; 2016. p. 1105-14.

25. Wang D, Cui P, Zhu W. Structural deep network embedding. In: ACM SIGKDD International Conference on Knowledge Discovery and Data Mining; 2016. p. 1225-34.

26. Fassan M, Saraggi D, Balsamo L, Cascione L, Castoro C, Coati I, Bernard MD, Farinati F, Guzzardo V, Valeri N. Let-7c down-regulation in helicobacter pylori -related gastric carcinogenesis. Oncotarget. 2016;7(4):4915-24.
27. Aslan D, Garde C, Nygaard MK, Helbo AS, Dimopoulos K, Hansen JW, Severinsen MT, Treppendahl MB, Sjø LD, Grønbæk K. Tumor suppressor microRNAs are downregulated in myelodysplastic syndrome with spliceosome mutations. Oncotarget. 2016;7(9):9951-63.

28. Kazuhiko B, Kumar S, Rachel S, Pranavkumar S, Reena M, Stephanie W, Vivek K, Eric D, Jegga AG, Bezerra JA. Integrative genomics identifies candidate microRNAs for pathogenesis of experimental biliary atresia. BMC Syst Biol. 2013;7(1):104.

29. Li WJ, Xie XX, Bai J, Wang C, Zhao L, Jiang DQ. Increased expression of miR1179 inhibits breast cancer cell metastasis by modulating notch signaling pathway and correlates with favorable prognosis. Eur Rev Med Pharmacol Sci. 2018;22(23):8374-82.

30. Merino MJ, Gil S, Macias CG, Lara K. The unknown microRNA expression of male breast cancer. Similarities and differences with female ductal carcinoma. Their role as tumor biomarker. J Cancer. 2018;9(3):450-9.

31. Boya X, Qin D, Hongjin H, Di W. miRCancer: a microRNA-cancer association database constructed by text mining on literature. Bioinformatics. 2013;29(5):638-44.

32. Phan B, Majid S, Ursu S, Semir DD, Nosrati M, Bezrookove V, Kashani-Sabet M, Dar AA. Tumor suppressor role of microRNA-1296 in triple-negative breast cancer. Oncotarget. 2016;7(15):19519-30.

33. Hu JY, Yi W, Zhang MY, Xu R, Zeng LS, Long XR, Zhou XM, Zheng XS, Kang $Y$, Wang HY. MicroRNA-711 is a prognostic factor for poor overall survival and has an oncogenic role in breast cancer. Oncol Lett. 2016;11(3):2155-63.

34. Song L, Dai Z, Zhang S, Zhang H, Liu C, Ma X, Liu D, Zan Y, Yin X. MicroRNA-1179 suppresses cell growth and invasion by targeting spermassociated antigen 5-mediated Akt signaling in human non-small cell lung cancer. Biochem Biophys Res Commun. 2018;504(1):164-170.

35. Jiang W, Tian Y, Jiang S, Liu S, Zhao X, Tian D. MicroRNA-376c suppresses non-small-cell lung cancer cell growth and invasion by targeting LRH-1-mediated Wnt signaling pathway. Biochem Biophys Res Commun. 2016;473(4):980-6.

36. Hu S, Yuan $Y$, Song Z, Yan D, Kong X. Expression profiles of microRNAs in drug-resistant non-small cell lung Cancer cell lines using microRNA sequencing. Cell Physiol Biochem. 2018;51(6):2509-22.

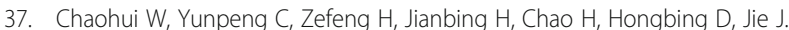
Serum levels of miR-19b and miR-146a as prognostic biomarkers for nonsmall cell lung cancer. Tohoku J Exp Med. 2014;232(2):85-95.

38. Mohan RD, Bibber B, Sinha G, Patel SA, Rameshwar P. MicroRNA in development and in the progression of cancer; 2014.

39. Moustafa AA, Ziada M, Elshaikh A, Datta A, Kim H, Moroz K, Srivastav S, Thomas R, Silberstein JL, Moparty K, et al. Identification of microRNA signature and potential pathway targets in prostate cancer. Exp Biol Med. 2017;242(5):536-46.

40. Stuopelyte K, Daniunaite K, Jankevicius F, Jarmalaite S. Detection of miRNAs in urine of prostate cancer patients. Medicina. 2016;52(2):116-24.

41. Ping X, Ke H, Maozu G, Yahong G, Jinbao L, Jian D, Yong L, Qiguo D, Jin L, Zhixia $T$ et al: The top 50 prostatic neoplasms-related miRNA candidates; 2013.

42. Yang $L$, Chengxiang $Q$, Jian $T$, Bin $G$, Jichun $Y$, Tianzi J, Qinghua C. HMDD V2.0: a database for experimentally supported human microRNA and disease associations. Nucleic Acids Res. 2014;42(Database issue):D1070.

43. Yang Z, Ren F, Liu C, He S, Sun G, Gao Q, Yao L, Zhang Y, Miao R, Cao Y. dbDEMC: a database of differentially expressed miRNAs in human cancers. BMC Genomics. 2010;11(Suppl 4):1-8.

44. Jiang Q, Wang Y, Hao Y, Juan L, Teng M, Zhang X, Li M, Wang G, Liu Y. miR2Disease: a manually curated database for microRNA deregulation in human disease. Nucleic Acids Res. 2009;37(1):D98-104.

45. Li Y, Qiu C, Tu J, Geng B, Yang J, Jiang T, Cui Q. HMDD v2.0: a database for experimentally supported human microRNA and disease associations. Nucleic Acids Res. 2014;42(Database issue):D1070.

46. Kozomara A, Griffiths-Jones S. miRBase: integrating microRNA annotation and deep-sequencing data. Nucleic Acids Res. 2011;39(suppl_1):D152-7.

47. Wen Z, Liu X, Chen Y, Wu W, Li X. Feature-derived graph regularized matrix factorization for predicting drug side effects. Neurocomputing. 2018;287:154-162.

48. Wen Z, Xiang Y, Feng H, Ruoqi L, Yanlin C, Chunyang R. Predicting drugdisease associations and their therapeutic function based on the drug-disease association bipartite network. Methods. 2018;145:51-59.

49. Wen Z, Xiang Y, Weiran L, Wenjian W, Ruoqi L, Feng H, Feng L. Predicting drug-disease associations by using similarity constrained matrix factorization. Bmc Bioinformatics. 2018;19(1):233. 
50. Zhang W, Chen Y, Li D, Yue X. Manifold regularized matrix factorization for drug-drug interaction prediction. J Biomed Inform. 2018;88:90-97.

51. Zhang W, Jing K, Huang F, Chen Y, Li B, Li J, Gong J. SFLLN: a sparse feature learning ensemble method with linear neighborhood regularization for predicting drug-drug interactions. Inf Sci. 2019;497:189-201.

52. Zhang W, Yu C, Wang X, Liu F. Predicting CircRNA-disease associations through linear neighborhood label propagation method. IEEE Access. 2019;7:83474-83.

53. Zhang W, Yue X, Tang G, Wu W, Huang F, Zhang X, loshikhes I. SFPEL-LPI: sequence-based feature projection ensemble learning for predicting LncRNA-protein interactions. PLoS Comput Biol. 2018;14(12):e1006616.

54. Wang D, Cui P, Zhu W. Structural Deep Network Embedding; 2016. p. 1225-34.

55. Goyal P, Ferrara E. Graph embedding techniques, applications, and performance: a survey. Knowl-Based Syst. 2018;151:78-94.

56. Elsevier. International Journal of Approximate Reasoning. Mathware Soft Comput. 2012;53(1):17-29.

57. Breiman L. Random forests. Mach Learn. 2001:45(1):5-32.

58. Chen C, Breiman L. Using random forest to learn imbalanced data; 2004

59. Taherzadeh G, Zhou Y, Liew AW, Yang Y. Structure-based prediction of protein- peptide binding regions using random Forest. Bioinformatics. 2018;34(3):477-84.

\section{Publisher's Note}

Springer Nature remains neutral with regard to jurisdictional claims in published maps and institutional affiliations.

Ready to submit your research? Choose BMC and benefit from:

- fast, convenient online submission

- thorough peer review by experienced researchers in your field

- rapid publication on acceptance

- support for research data, including large and complex data types

- gold Open Access which fosters wider collaboration and increased citations

- maximum visibility for your research: over $100 \mathrm{M}$ website views per year

At BMC, research is always in progress.

Learn more biomedcentral.com/submissions 